\title{
CONTINUIDADES Y CAMBIOS EN LAS OPINIONES Y ACTITUDES DE LOS PROFESORES DE SECUNDARIA DE ARGENTINA EN EL PERÍODO 2000-2010
}

\author{
LEANDRO BOTTINELLI*
}

\begin{abstract}
RESUMEN: El nivel de enseñanza secundario en Argentina está atravesando un período de debates y transformaciones. Sus profesores no están ajenos a este proceso. Mediante dos encuestas de alcance nacional realizadas en los años 2000 y 2010 se estudiaron los cambios en sus opiniones y actitudes frente a temas tan diversos como los problemas pedagógicos, las aspiraciones profesionales y las percepciones de su condición social, entre otros. Los resultados dan cuenta de una subjetividad docente que también se halla en proceso de transformación.
\end{abstract}

Palabras clave: Educación secundaria. Reforma educativa. Profesión docente. Docentes y estructura social.

\section{Changes And continuity in Argentine secondary teachers' OPINIONS AND ATTITUDES BETWEEN 2000 AND 2010}

ABSTRACT: In Argentina, secondary school is going through a period of debates and transformations. Teachers have played an import role in this process. Two national surveys carried out in 2000 and 2010 studied how their opinions and attitudes about pedagogical problems, career aspirations and perceptions of social status, among others, have changed. The results indicate that teachers' identity is also changing.

Key words: Secondary education. Education reform. Teaching profession. Professors and social structure.

\section{Changements et CONTINUITÉS DANS LES OpINIONS ET ATTITUdeS}

DES ENSEIGNANTS ARGENTINS DU SECONDAIRE ENTRE 2000 ET 2010

RÉSUMÉ: En Argentine, l'éducation secondaire traverse une période de débats et de changements auxquels les professeurs sont loin d'être étrangers. Deux enquêtes nationales réalisées en 2000 et 2010 révèlent leurs changements d'opinions et d'attitude pour ce qui est des problèmes pédagogiques, de leurs aspirations professionnelles et de la perception qu'ils ont de leur condition

* Sociólogo, con estudios de maestría en Estadística y docente en la Universidad de Buenos Aires. E-mail: leandrobottinelli@yahoo.com.ar 
sociale, entre autres. Les résultats montrent que leur subjectivité enseignante est également en pleine transformation.

Mots-clés: Éducation secondaire. Reforme de l'éducation. Profession enseignante. Enseignants et structure sociale.

\section{Presentación}

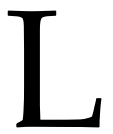

a enseñanza secundaria de Argentina está siendo objeto de un debate profundo desde hace algunos años. La enumeración de los desafíos a los que se enfrenta el nivel puede ser extensa y resultar reiterativa. Baste recordar aquí las consecuencias que acarrea la obligatoriedad desde el año 2006 (Argentina, 2006b) así como las dificultades que enfrenta un sub-sistema que se originó como instancia selectiva y de tránsito hacia los estudios superiores y que, desde hace unas décadas, debe transformarse para educar a todos y a todas y para una gran diversidad de propósitos (Tenti Fanfani, 2010).

Como todo hecho social, la escuela se encuentra atravesada por diferentes significados. Los agentes que la construyen cotidianamente tienen ideas y creencias particulares, a veces divergentes, sobre este objeto social, su misión y sus necesidades. La reflexión que vamos a compartir se ubica en una tradición que reconoce la necesidad de comprender qué piensan y sienten los agentes sociales como requisito indispensable para promover transformaciones. Los y las docentes tal vez sean los principales "productores de escuela" en su trabajo cotidiano en las aulas. Conocer su subjetividad, reconstruir sus perspectivas, sus temores, caracterizar las estructuras de percepción a través de las que decodifican y construyen la realidad, es un paso necesario en el camino de entender por qué las "cosas" de la educación son como son y qué se podría hacer para que fueran de otra manera.

Este artículo propone una reflexión a la luz de los resultados de dos encuestas de alcance nacional, circunscriptas al ámbito urbano, que fueron aplicadas a docentes de escuela secundaria en los años 2000 y 2010. Sus objetivos fueron conocer de manera general un conjunto extenso de características subjetivas de este colectivo. Los estudios fueron diseñados e implementados por el Instituto Internacional de Planeamiento de la Educación (IIPE-unesco), Sede Regional Buenos Aires, bajo la coordinación de Emilio Tenti Fanfani y en el marco de un programa de investigación más amplio sobre la profesión docente.

Los resultados que se presentan se enfocan en los cambios registrados en las opiniones y actitudes de los y las profesores/as en la década que media entre 2000 y 2010 y recorren dimensiones pedagógicas, profesionales y sociales. Así, se analizan los cambios en las opiniones respecto de los fines que debe perseguir la educación; la percepción de problemas en la tarea cotidiana de enseñar; la modificación de las 
actitudes de los profesores frente a las nuevas tecnologías de información y comunicación (en adelante TIC); las variaciones en sus aspiraciones profesionales para los próximos años; y los cambios en la percepción de la propia condición social.

\section{Algunas consideraciones metodológicas}

Los dos estudios que dan origen a este artículo fueron concebidos con un carácter exploratorio y para abarcar una variada gama de dimensiones de análisis. Los resultados ofrecen un panorama amplio sobre opiniones y actitudes pero con limitaciones respecto de la comprensión de los sentidos implicados en ellas.

El diseño del cuestionario tomó como punto de partida las dimensiones de análisis del estudio. ${ }^{1}$ Se consideraron también las observaciones y comentarios recibidos en las entrevistas con directores de escuela y en los grupos focales con docentes para ajustar las preguntas e ítems de respuesta utilizados en el cuestionario. Se instrumentaron tres pruebas piloto para trabajar la claridad, consistencia, orden de las preguntas, variabilidad y validez de los indicadores. El cuestionario aplicado en 2010 reiteró un conjunto de preguntas que permitieran establecer una comparación con los resultados del año 2000 e incluyó algunas nuevas para captar opiniones sobre nuevas problemáticas emergentes.

El universo de estudio estuvo conformado por docentes de secundaria que trabajan frente a alumnos en escuelas urbanas de todo el país, tanto en el sector estatal como en el privado. En la encuesta del 2000, los docentes de secundaria correspondían al ciclo de la EGB3 y al Polimodal; en la del 2010 a la escuela secundaria, fuera ésta de 5 o 6 años de duración (Cuadro 1).

La muestra de docentes de ambas investigaciones tuvo un carácter probabilístico que permitió inferir resultados para el universo de estudio. Además, el diseño muestral estratificado por nivel de enseñanza posibilitó desagregar datos para profesores de secundaria. La selección de casos se realizó en etapas sucesivas: localidades, escuelas y docentes. La encuesta se aplicó de manera auto-administrada entre el 28 de agosto y el 15 de septiembre en el año 2000, y entre el 26 de mayo y el 18 de junio en el 2010. La cantidad final de docentes de secundaria encuestados fue de 1.164 y 1.264 respectivamente.

Debido al origen muestral de los datos, los resultados contienen un cierto margen de error. Para el caso de una proporción del 50\%, el error es de 2,9\% en las estimaciones del año 2000 y de 2,8\% en las de 2010, en ambos casos para un nivel de confianza del $95 \%$. A partir de estos valores de error, se calcularon los límites superior (valor máximo) e inferior (valor mínimo) de cada estimación puntual (CepedaCuervo et al., 2008, p. 213-215). Las variaciones observadas entre ambas encuestas 
fueron consideradas como "estadísticamente significativas" (es decir, no debidas al error de muestreo) cuando el límite superior del porcentaje más bajo y el límite inferior del porcentaje más alto, no se encontraban superpuestos. En cada cuadro con datos se indica si las variaciones observadas entre ambos estudios resultan estadísticamente significativas.

\section{Cuadro 1}

Equivalencia de niveles y años de estudio entre las estructuras académicas: tradicional y prescriptos por la Ley Federal de Educación y la Ley de Educación Nacional.*

\begin{tabular}{|c|c|c|c|c|c|c|c|}
\hline \multirow{2}{*}{\multicolumn{2}{|c|}{ Tradicional }} & \multirow{2}{*}{\multicolumn{2}{|c|}{ LFE 1993}} & \multicolumn{4}{|c|}{ LEN 2006 (Actual) } \\
\hline & & & & \multicolumn{2}{|c|}{7 Primario - 5 Secundario } & \multicolumn{2}{|c|}{6 Primario - 6 Secundario } \\
\hline Nivel & Año & Nivel & Año & Nivel & Año & Nivel & Año \\
\hline \multirow{7}{*}{ Primario } & 1 & \multirow{6}{*}{ EGB 1 y 2} & 1 & \multirow{7}{*}{ Primario } & 1 & \multirow{6}{*}{ Primario } & 1 \\
\hline & 2 & & 2 & & 2 & & 2 \\
\hline & 3 & & 3 & & 3 & & 3 \\
\hline & 4 & & 4 & & 4 & & 4 \\
\hline & 5 & & 5 & & 5 & & 5 \\
\hline & 6 & & 6 & & 6 & & 6 \\
\hline & 7 & \multirow{4}{*}{ EGB 3} & 7 & & 7 & \multirow{6}{*}{ Secundario } & 1 \\
\hline \multirow{5}{*}{ Secundario } & 1 & & 8 & & 1 & & 2 \\
\hline & 2 & & 9 & & 2 & & 3 \\
\hline & 3 & & 1 & Secundario & 3 & & 4 \\
\hline & 4 & \multirow[t]{2}{*}{ Polimodal } & 2 & & 4 & & 5 \\
\hline & 5 & & 3 & & 5 & & 6 \\
\hline
\end{tabular}

Fuente: Cappellacci et al., 2011, p. 8.

* Nota: las celdas sombreadas corresponden a los universos de estudio de cada encuesta.

Una última consideración metodológica está relacionada con el contexto de aplicación de cada encuesta. El relevamiento del 2000 se inscribe en el primer año del gobierno de la Alianza (coalición entre radicales y frepasistas), gestión que había sucedido en 1999 a la década de reformas de corte neoliberal de la administración Menem. El período corresponde a una fase de recesión económica que, si bien no alcanzaba aún el dramatismo que adquirió a fines de 2001, estaba marcado por el octavo trimestre sin crecimiento en el nivel de actividad. El contexto de aplicación de la encuesta en 2010 es bien distinto ya que se ubica en una fase larga de crecimiento económico iniciada a fines de 2002 y sólo interrumpida por la breve recesión del año 2009 (Argentina, 2011c). Es un período marcado por mejoras en las expectativas económicas de la población y, en gran medida, por el repunte de las perspectivas políticas futuras de la gestión de Cristina Kirchner. El señalamiento realizado debe tenerse en cuenta al analizar los resultados ya que, parte del incremento en las percepciones positivas (por ejemplo, de la propia condición social 
de los y las docentes) podría ser leído en un contexto de mejora socio-económica general que trasciende el campo de lo estrictamente educativo.

\section{La educación secundaria de Argentina y sus docentes}

La educación secundaria en Argentina comienza, según la provincia, luego de 6 o 7 años de educación primaria. La estructura actual del nivel es el resultado de dos reformas sucesivas implementadas en las últimas décadas a partir de la sanción de la Ley Federal de Educación n. 24.195 (Argentina, 1993), en adelante LFE, y de la Ley de Educación Nacional n. 26.206 (Argentina, 2006b), en adelante LEN.

La extensión de la obligatoriedad hasta el último año de la secundaria establecido por la LEN de 2006, está suponiendo un impacto de envergadura para el subsistema. Aún cuando su cobertura es alta desde hace algunos años, ${ }^{2}$ dista aún del techo al que se ha acercado el nivel primario, y se caracteriza por una considerable heterogeneidad territorial y social (Cappellacci et al., 2011). A partir de los lineamientos para la reforma de la escuela secundaria que define la LEN en su artículo 32 , se han instrumentado diversas acciones apuntadas a la reinclusión escolar de jóvenes, al fortalecimiento institucional y a la reforma curricular (Argentina, 2011a), cuyos impactos son aún objeto de debate.

El colectivo docente en Argentina alcanza los 825.250 integrantes según el último dato oficial disponible correspondiente al año 2004. El 53,3\% de ellos se desempeñan en el nivel secundario (Argentina, 2006c). Si se tiene en cuenta que el censo releva cuatro niveles de enseñanza (inicial, primario, secundario y superior no universitario), se comprende que se trata de un colectivo docente muy extendido. Por otra parte, y en comparación con los maestros de primaria, los y las profesores/as de secundaria son un grupo menos feminizado, con un promedio de edad superior, con un porcentaje menor con formación pedagógica y con padres con un nivel educativo mayor (Dirie, 2007).

\section{Opinión sobre los fines que debe perseguir la educación}

En el contexto de la denominada Tercera Revolución Educativa (Esteve, 2006) las demandas sobre la escuela se han diversificado. Ya no se espera solo que "enseñe", sino que también lo haga de manera interesante, que contenga, que acompañe a las familias, que sea participativa, que distribuya alimentos, entre otras funciones (Dussel, 2006).

Con el objetivo de conocer la forma en que los docentes de secundaria jerarquizan estas múltiples funciones, se solicitó su opinión respecto de cuáles son los 
fines más importantes que debe perseguir la educación. Sobre un listado de nueve opciones de respuesta se les pidió señalar los dos fines más importantes y los dos menos importantes.

\section{Cuadro 2}

Fines más importantes que debe perseguir la educación según los docentes de secundaria.

Años 2000 y 2010. Porcentaje de encuestados que seleccionó cada ítem. ${ }^{3}$

\begin{tabular}{|l|r|r|r|r|r|}
\hline \multicolumn{1}{|c|}{ Items de respuesta } & 2000 & 2010 & $\begin{array}{c}\text { Dif. } \\
\text { pp. }\end{array}$ & \multicolumn{1}{c|}{$\begin{array}{c}\text { Dif. } \\
\%\end{array}$} & $\begin{array}{c}\text { Var. } \\
\text { Signif. }\end{array}$ \\
\hline Desarrollar la creatividad y el espíritu crítico & 63,0 & 52,9 & $-10,0$ & $-15,9$ & Sí \\
Preparar para la vida en sociedad & 37,2 & 45,6 & 8,4 & 22,7 & Sí \\
Transmitir conocimientos actualizados y relevantes & 30,3 & 23,4 & $-6,9$ & $-22,9$ & Sí \\
Transmitir valores morales & 23,2 & 28,6 & 5,4 & 23,4 & Sí \\
Formar para el trabajo & 16,4 & 14,8 & $-1,6$ & $-9,7$ & No \\
Promover la integración de los grupos sociales más & 15,9 & 12,9 & $-3,1$ & $-19,4$ & No \\
postergados & 6,0 & 11,1 & 5,1 & 83,9 & Sí \\
Crear hábitos de comportamiento & 3,3 & 8,9 & 5,6 & 168,0 & Sí \\
Proporcionar conocimientos mínimos & 1,9 & 1,3 & $-0,6$ & $-32,6$ & No \\
Seleccionar a los sujetos más capacitados & & &
\end{tabular}

Fuente: Encuesta Nacional a Docentes 2000 y 2010 (IIPE-UnEsCo, Sede Regional Buenos Aires).

En ambas encuestas (Cuadro 2) las respuestas priorizaron como fin más importante el de desarrollar la creatividad y el espíritu crítico, aunque el ítem perdió participación relativa entre observaciones. En segundo lugar, y también en ambos relevamientos, se ubicó la opción preparar para la vida en sociedad. Contrariamente a lo sucedido con el ítem precedente, esta opción ganó importancia en las respuestas pasando de un $37,2 \%$ en el 2000 a un $45,6 \%$ en 2010.

Un conjunto de ítems que figura entre los más elegidos y que incrementaron su participación son los que aluden a la dimensión "socializadora" de la educación como preparar para la vida en sociedad, crear hábitos de comportamiento y transmitir valores morales. Por otra parte, expresiones asociadas al componente cognoscitivo como desarrollar la creatividad y el espíritu crítico y transmitir conocimientos actualizados y relevantes, perdieron posiciones relativas en las respuestas entre ambos relevamientos. Se registra como excepción a este punto el ítem proporcionar conocimientos mínimos 
que obtuvo menos del 10\% de las respuestas en ambos relevamientos. La dimensión vinculada a la equidad educativa, representada por el ítem promover la integración de los grupos sociales más postergados, también perdió consideración en las respuestas de los encuestados, aunque la variación no resulte estadísticamente significativa.

La mayor importancia relativa que adquieren las respuestas asociadas con hábitos, valores, vida en sociedad, invita a pensar en una preocupación emergente de los profesores por las funciones socializadoras y de integración históricamente asociadas más a la educación primaria que a la secundaria. Las crecientes dificultades que expresan los docentes para "ordenar la clase" antes de comenzar a enseñar y para convocar la atención de los estudiantes (Dussel, 2006) resuenan también en esta jerarquización de los ítems asociados a los comportamientos y a la vida grupal. El predomino de estas preocupaciones podría estar relegando en las respuestas las funciones relacionadas con la trasmisión de conocimientos (Meo, 2010).

Hasta aquí el análisis realizado se enfoca en los fines que los encuestados señalaron como "más importantes". Si nos detenemos en los fines "menos importantes", el listado de respuestas está encabezado por el ítem seleccionar los sujetos más capacitados, el cual fue seleccionado por el $69,2 \%$ de los encuestados en 2000 y por el 75,3\% en 2010. La expresión remite, como puede observarse, a un concepto tan tradicional como elitista de la educación. En cierta medida, el pasado de la escuela media en Argentina estuvo marcado en su desenvolvimiento por un criterio de selección según el cual el "fracaso" y la "deserción" eran fenómenos habituales y hasta esperados (Tenti Fanfani, 2010, p. 42). Afortunadamente el enfoque del derecho a la educación ha transformado aquella visión, más allá de que muchas prácticas sigan reproduciendo lógicas de selección asociadas con las condiciones sociales.

\section{Problemas percibidos en la tarea de enseñar}

Para conocer el modo en que se ordenan los problemas que experimentan los y las docentes en su tarea como educadores el cuestionario presentaba trece ítems relacionados con problemas típicos que fueron expresados por los docentes en instancias de entrevistas y grupos focales. Dichos ítems corresponden a determinadas dimensiones del proceso de enseñanza-aprendizaje como la organizativa, la pedagógica, la vincular y la contextual.

Antes de analizar la información, debe señalarse que en esta pregunta no se indicó a los encuestados una cantidad máxima de respuestas posibles, lo que redundó en un total de problemas señalados diferente en cada relevamiento (Cuadro 3). En el correspondiente al 2000 se registró un promedio de problemas consignados por encuestado mayor que en el 2010: 4,6 en el primer caso, 3,8 en el segundo. 
Podría interpretarse que esta diferencia no es casual y que puede obedecer o bien, a la concentración de las preocupaciones en una menor cantidad de problemas, o bien a un contexto educativo y/o social que se vivencia como menos problemático. Probablemente un marco de reforma educativa profunda que aún estaba presente en el año 2000 produjera ese plus de problemas identificados merced a la conmoción de ciertos marcos de referencia que orientaban la práctica docente. Otra hipótesis a considerar se relaciona con un relativo acostumbramiento a cambios producidos por esa reestructuración inaugural de los noventa que tornó menos conflictiva la vivencia de los cambios correspondientes a la siguiente década.

\section{Cuadro 3}

Problemas identificados por los docentes de secundaria. Años 2000 y 2010. Porcentaje de encuestados que seleccionó cada ítem.

\begin{tabular}{|l|r|r|r|r|r|}
\hline \multicolumn{1}{|c|}{ Items de respuesta } & 2000 & 2010 & $\begin{array}{c}\text { Dif. } \\
\text { pp. }\end{array}$ & \multicolumn{1}{c|}{$\begin{array}{c}\text { Dif. } \\
\%\end{array}$} & $\begin{array}{c}\text { Var. } \\
\text { signif. }\end{array}$ \\
\hline $\begin{array}{l}\text { El tiempo disponible para corregir evaluaciones, cuadernos, } \\
\text { etc. }\end{array}$ & 68,8 & 60,1 & $-8,7$ & $-12,7$ & Sí \\
$\begin{array}{l}\text { La falta de definiciones y objetivos claros sobre lo que hay } \\
\text { que hacer }\end{array}$ & 63,8 & 35,8 & $-28,0$ & $-43,8$ & Sí \\
El tiempo disponible para el desarrollo de las tareas & 61,0 & 42,7 & $-18,3$ & $-30,0$ & Sí \\
La disponibilidad de ámbitos de asesoramiento y & 56,8 & 48,8 & $-8,0$ & $-14,1$ & Sí \\
supervisión & 52,2 & 40,3 & $-11,9$ & $-22,8$ & Sí \\
Las características sociales de los alumnos & 35,6 & 37,3 & 1,8 & 5,0 & Sí \\
La relación con los padres & 33,0 & 35,5 & 2,6 & 7,8 & Sí \\
Manejar la disciplina en clase & 25,1 & 15,5 & $-9,6$ & $-38,3$ & Sí \\
Las formas de planificar & 23,8 & 19,7 & $-4,0$ & $-16,9$ & Sí \\
Evaluar & 12,1 & 8,8 & $-3,3$ & $-27,1$ & Sí \\
El dominio de los nuevos contenidos & 11,0 & 14,2 & 3,1 & 28,6 & Sí \\
Organizar el trabajo en clase & 9,7 & 11,0 & 1,3 & 13,5 & No \\
El trabajo con los colegas & 9,6 & 8,4 & $-1,2$ & $-12,7$ & No \\
La relación con los directivos & 4,6 & 3,8 & $-0,8$ & $-17,4$ & Sí \\
Promedio de problemas señalados por cada encuestado & &
\end{tabular}

Fuente: Encuesta Nacional a Docentes 2000 y 2010 (IIPE-UnEsCo, Sede Regional Buenos Aires).

Los problemas que se ubican entre los más señalados en el año 2000 son $e l$ tiempo disponible para corregir evaluaciones y cuadernos, la falta de definiciones y objetivos 
claros sobre lo que hay que hacer, y el tiempo disponible para el desarrollo de las tareas. Cada uno de los tres ítems fue señalado por más del $60 \%$ de los encuestados. Como se observa, se trata de problemas relacionados en gran medida con el uso del tiempo. Es posible que esta priorización esté expresando una demanda frecuente de los profesores respecto del no reconocimiento de las tareas que se realizan fuera del establecimiento escolar. También, y como ha sido señalado por otros estudios, puede estar operando un malestar frente a nuevas tareas administrativas no relacionadas directamente con la enseñanza y que el nuevo contexto requiere al docente (Avalos, 2010).

En el año 2010 se observa la persistencia de los problemas asociados al tiempo disponible pero con una menor importancia asignada en particular al tiempo disponible para el desarrollo de las tareas. Por otra parte, la falta de definiciones y objetivos sobre lo que hay que hacer es un aspecto que es señalado en mucho menor medida que en el año 2000. Una de las hipótesis a considerar para dar cuenta de esta situación podría remitir nuevamente al contexto de reforma educativa presente aún en el año 2000 y que habría provocado cambios en los marcos de referencia del trabajo docente.

Mientras la tendencia general en esta pregunta es que los y las docentes señalen una cantidad menor de problemas en su tarea cotidiana de enseñar con respecto al año 2000, hay sin embargo algunas excepciones correspondientes a la dimensión vincular. Los ítems relacionados con los destinatarios de la labor docente como relación con los padres y manejar la disciplina en clase, son algunos de ellos. Otros como organizar el trabajo en clase y el trabajo con los colegas, que en parte remiten a lo vincular y en parte a otras dimensiones, atrajeron también una mayor proporción de respuestas en el año 2010. Al recalcular los porcentajes de respuesta de estos ítems sobre una misma base para comparar exclusivamente la importancia relativa de los problemas en cada medición, se verifica que, con excepción del ítem trabajo con colegas, los otros tres problemas asociados a lo vincular experimentan un incremento estadísticamente significativo. En definitiva, las preocupaciones asociadas con la interacción con los destinatarios de la enseñanza (los estudiantes y sus familias), si bien no están entre las más señaladas, son las que más presencia ganaron en la última década.

\section{Actitudes frente a las TIC en el marco de la enseñanza}

Desde una perspectiva relacional, puede considerarse que resulta erróneo plantear si las tecnologías de información y comunicación son buenas o malas para el proceso de enseñanza-aprendizaje ya que lo importante es definir cómo, quién y con qué fines se las utiliza (Burbules y Callister, 2006). Sin embargo, desde el punto de vista de los actores, la incorporación de las TIC en la enseñanza genera una gama de sentimientos encontrados que pueden transformarse en resistencias para 
su adecuada incorporación y apropiación. Al respecto, se ofreció a la consideración de los docentes un listado de expresiones sobre tic elaboradas a partir del trabajo en grupos focales. Se trataba de seis frases que expresaban ideas positivas y negativas - tres en cada caso - sobre las consecuencias de la incorporación de las tic en la tarea pedagógica.

\section{Cuadro 4}

Acuerdo de los docentes de secundaria con afirmaciones sobre el impacto de las tic en la enseñanza. Años 2000 y 2010. Porcentaje de encuestados que manifestó acuerdo con cada ítem.

\begin{tabular}{|l|c|c|c|c|c|}
\hline \multicolumn{1}{|c|}{ Items de respuesta } & 2000 & 2010 & $\begin{array}{c}\text { Dif. } \\
\text { pp. }\end{array}$ & $\begin{array}{c}\text { Dif. } \\
\%\end{array}$ & $\begin{array}{c}\text { Var. } \\
\text { signif. }\end{array}$ \\
\hline $\begin{array}{l}\text { Van a ampliar las oportunidades de acceso al conocimiento } \\
\text { por parte de los alumnos }\end{array}$ & 82,9 & 76,4 & $-3,9$ & $-7,8$ & Sí \\
$\begin{array}{l}\text { Son recursos que facilitarán la tarea de los docentes en las } \\
\text { aulas }\end{array}$ & 81,3 & 71,7 & $-6,9$ & $-11,8$ & Sí \\
$\begin{array}{l}\text { Permitirán mejorar la calidad de la educación y el } \\
\text { aprendizaje }\end{array}$ & 68,8 & 59,6 & $-8,0$ & $-13,3$ & Sí \\
$\begin{array}{l}\text { Van a alentar el facilismo de los alumnos } \\
\begin{array}{l}\text { Van a contribuir a deshumanizar la enseñanza y las } \\
\text { instituciones pedagógicas }\end{array}\end{array}$ & 31,3 & 36,7 & 5,9 & 17,3 & Sí \\
$\begin{array}{l}\text { Van a reemplazar parcialmente el trabajo de los docentes } \\
\text { en el aula }\end{array}$ & 18,6 & 14,9 & $-4,4$ & $-20,1$ & No \\
\hline
\end{tabular}

Fuente: Encuesta Nacional a Docentes 2000 y 2010 (IIPE-UNESCO, Sede Regional Buenos Aires).

La interpretación que se desprende de los datos de ambas encuestas (Cuadro 4) es que, en general, existe una actitud positiva respecto de la incorporación de las TIC en la enseñanza. Los ítems que expresan las posiciones más favorables fueron suscriptos por entre el 59,6\% y el 82,9\% de los entrevistados. Por el contrario, las expresiones negativas concitaron adhesiones entre el $14,9 \%$ y el $36,9 \%$.

Más allá de la mirada mayoritariamente positiva, existe un núcleo constituido por una cuarta parte de los docentes para los que las Tic, o la forma en que ellas están siendo incorporadas en la enseñanza, son motivo de desconfianza, situación que no ha variado sustancialmente en los últimos diez años. Este grupo de docentes tiene una mayor representación en la región de Cuyo y del Noroeste, y también entre aquellos de menor nivel socio-económico.

El análisis de los resultados para cada una de las expresiones arroja elementos adicionales para la reflexión. El problema del "facilismo" es, de los tres ítems que 
hemos denominado como negativos, el que más se incrementó. Por otra parte, las tres frases que expresan las visiones positivas, se ordenan del mismo modo en ambos relevamientos. En primer lugar se posiciona la idea de que las TIC van a ampliar las posibilidades de "acceso al conocimiento" por parte de los alumnos; en segundo lugar que resultan un "recurso" que hace más fácil la tarea docente en el aula, y en tercer lugar (siempre con el acuerdo del $60 \%$ o más de los docentes) que habilitarán una mejora en la calidad de la educación.

Más allá de que el porcentaje de encuestados que concuerda con los ítems que expresan la visión positiva sigue siendo alto, es sintomático el hecho de que disminuyan en los tres casos. En paralelo a este dato de la subjetividad, la encuesta permitió saber que los profesores han incrementado de manera considerable su acceso a tic. Como ejemplo puede mencionarse que mientras en el 2000 un 65,9\% tenía computadora en su casa, en el 2010 el porcentaje se eleva al 88,3\%. En cuanto al correo electrónico, su uso frecuente pasó del $23,2 \%$ al $78,4 \%$ entre ambas mediciones. Por otro lado, si bien el grado en que los docentes ha incorporado las Tic para el uso pedagógico solo se midió en la encuesta del 2010, es un dato significativo la extensión que ha adquirido el fenómeno: el 85,6\% las utiliza para preparar clases; el 76,1\% para solicitar trabajos a los alumnos; el 66,1\% para sistematizar información sobre notas; y el $40,0 \%$ para dar clases. Esta incorporación de las tic en la vida personal y profesional de los docentes, podría contribuir a explicar una concepción más compleja y completa y, por tanto, algo menos optimista, de las oportunidades que ofrecen para la enseñanza. Probablemente en el año 2000 las TIC resultaban aún una promesa para la gran mayoría, una gran oportunidad que solo algunos habían experimentado en el aula o en su casa. Diez años después las actitudes tienen un sustento en la propia experiencia que, probablemente, se traduzca en una consideración tanto de las oportunidades como de los desafíos que las tic generan.

\section{Aspiraciones profesionales}

La configuración de los proyectos laborales y profesionales de los docentes obedece a múltiples factores. En su determinación se cruzan tanto motivaciones personales y familiares, como las oportunidades de desarrollo que ofrece el propio sistema educativo y las alternativas de ocupación existentes fuera del ámbito de la educación. Desde el punto de vista del sistema educativo, una de las preocupaciones principales es cómo atraer y retener buenos docentes combinando buenas condiciones salariales y otras no salariales (Morduchowicz, 2004). Para definir cómo opera este segundo tipo de condiciones es necesario estudiar qué priorizan los docentes al momento de tomar decisiones respecto de su carrera profesional y cuáles son las aspiraciones profesionales para el futuro cercano. 
Para reconocer cómo se ordenan los proyectos profesionales futuros de los profesores de secundaria se incluyó en los cuestionarios un ítem en el que se los consultaba sobre sus "aspiraciones laborales y profesionales para los próximos años". Desafortunadamente el diseño de las opciones de respuesta de la encuesta del 2000 no permitió captar algunas opciones clave. Por ese motivo, el listado de respuestas de la encuesta de 2010 fue ampliado para abarcar una mayor diversidad al precio de perder algo de comparabilidad.

Un interrogante que se buscaba responder era en qué medida los profesores querían mantenerse en su puesto actual en el aula, dado los efectos que producen los años transcurridos en un contexto de aula crecientemente complejo, en la interacción con los estudiantes y sus familias.

\section{Cuadro 5}

Aspiraciones laborales y profesionales para los próximos años. Años 2000 y 2010. Porcentaje de encuestados que seleccionó cada ítem.

\begin{tabular}{|l|r|r|r|r|c|}
\hline \multicolumn{1}{|c|}{ Items de respuesta } & 2000 & 2010 & \multicolumn{1}{|c|}{$\begin{array}{c}\text { Dif. } \\
\text { pp. }\end{array}$} & $\begin{array}{c}\text { Dif. } \\
\%\end{array}$ & $\begin{array}{c}\text { Var. } \\
\text { signif. }\end{array}$ \\
\hline Seguir en su puesto actual & 46,7 & 34,9 & $-11,8$ & $-25,3$ & Sí \\
$\begin{array}{l}\text { Realizar otra actividad profesional en el ámbito } \\
\text { educativo }\end{array}$ & 20,3 & 10,7 & $-9,6$ & $-47,2$ & Sí \\
Ocupar cargos de dirección y gestión & 18,1 & 11,9 & $-6,1$ & $-34,0$ & Sí \\
Ascender de categoría sin dejar la sala de clase & & 22,5 & & - & - \\
Dedicarse a otra ocupación & 6,4 & 5,8 & $-0,6$ & $-9,3$ & No \\
Lo mismo que hace ahora pero en otra institución & 1,7 & 2,2 & 0,6 & 34,4 & No \\
Jubilarse & & 8,2 & & & - \\
Otra & & 0,5 & & & - \\
No sabe & 6,8 & 3,1 & $-3,7$ & $-54,2$ & No \\
\hline Total & 100,0 & 100,0 & & & \\
\hline
\end{tabular}

Fuente: Encuesta Nacional a Docentes 2000 y 2010 (IIPE-UnEsCo, Sede Regional Buenos Aires).

En la encuesta del 2000 (Cuadro 5), solo el 46,7\% de los entrevistados tenía aspiraciones de seguir en su puesto actual. Realizar otra actividad profesional en el ámbito educativo $(20,3 \%)$ y ocupar cargos de dirección y gestión $(18,1 \%)$ eran las opciones que seguían en el ranking de respuestas. Completándose la lista con los que querían dedicarse a otra ocupación fuera del ámbito educativo (6,4\%), el mapa de 
opciones marcaba que casi la mitad de los profesores esperaba dejar el aula en los próximos años.

En los grupos focales realizados en la etapa de diseño del cuestionario del año 2010 algunos docentes manifestaron que aunque valoraban el trabajo en el aula con los adolescentes también tenían la necesidad de asumir otras responsabilidades, otros desafíos y de acceder a otro tipo de reconocimiento salarial. En función de este argumento se incluyó en la pregunta sobre aspiraciones el ítem ascender de categoría sin dejar la sala de clase.

Los resultados correspondientes al 2010 muestran que si se suman los valores correspondientes a seguir en su puesto actual $(34,9 \%)$ y ascender de categoría sin dejar la sala de clase (22,5\%), la proporción de docentes que espera seguir trabajando en el curso con los estudiantes alcanza el 57,4\%. Podría conjeturarse que el incremento de más de 10 puntos porcentuales con respecto a la encuesta anterior, se debe a un cambio en la percepción de la situación en las aulas, pero es más probable que se deba a la introducción de una variante de respuesta que habilita simultáneamente "ascender sin dejar el aula". Para casi un cuarto de los profesores $(22,5 \%)$ se trata de una alternativa atractiva pero que desafortunadamente no está prevista por una carrera magisterial que solo permite el ascenso dejando el trabajo en el aula (Morduchowicz, 2004).

\section{Percepción de la propia condición social}

La ocupación docente en Argentina ha sido considerada como perteneciente a los niveles intermedios de la estructura social (Germani, 2010; Tenti Fanfani, 2006), señalándose grados diversos de empobrecimiento, descenso social o proletarización de su condición en las décadas recientes (Donaire, 2009). Estudios de otros países han relevado y analizado cómo los docentes perciben que su profesión se encuentra subvalorada por la sociedad en el marco de las transformaciones que en las últimas décadas afectaron a la economía, la sociedad y los sistemas educativos (Avalos, 2010).

El cambio en la percepción de la propia condición social de los y las profesores/as en Argentina fue también objeto de análisis de las encuestas realizadas. Asumiendo las limitaciones que tiene un instrumento como el cuestionario estructurado para abordar un tema tan complejo, se solicitó a los docentes señalar su pertenencia a una de las siguientes categorías sociales: clase alta, media-alta, media, media-baja o baja.

La adscripción de las ocupaciones docentes a los estratos medios que señala parte importante de la literatura especializada, encuentra correspondencia en la 
autopercepción que los encuestados manifestaron (Cuadro 6). En ambos relevamientos, el ítem clase media fue la categoría modal con el 54,3\% de las respuestas en 2000 y el 64,6\% en 2010. La opción clase media baja fue la segunda más elegida en ambos relevamientos (36,1 y 23,4\% respectivamente) superando por lejos a su par opuesto superior (clase media alta).

\section{Cuadro 6}

Autopercepción de la clase social de los profesores.

Años 2000 y 2010. En Porcentaje.

\begin{tabular}{|l|c|c|c|c|c|}
\hline Items de respuesta & 2000 & 2010 & Dif. pp. & Dif. \% & Var. signif. \\
\hline Alta & 0,0 & 0,9 & 0,9 & - & Sí \\
Media alta & 2,0 & 5,9 & 3,9 & 195,0 & Sí \\
Media & 54,3 & 64,6 & 10,3 & 19,0 & Sí \\
Baja & 36,1 & 23,4 & $-12,7$ & $-35,2$ & Sí \\
No sabe & 3,2 & 1,5 & $-1,7$ & $-53,1$ & Sí \\
\hline Total & 4,4 & 3,8 & $-0,6$ & $-13,6$ & No \\
\hline
\end{tabular}

Fuente: Encuesta Nacional a Docentes 2000 y 2010 (IIPE-UnEsco, Sede Regional Buenos Aires).

El incremento que se verifica en la participación de la categoría modal entre ambas encuestas, se reitera en las dos categorías superiores de la escala (alta y mediaalta) generando un efecto de "ascenso social" de 14,6 puntos porcentuales a costa de los dos grados inferiores de la pirámide (baja y media-baja). Estos cambios configuran una estructura de base más estrecha con estratos medios más robustos.

Cuando se relaciona el mapa social que surge de la auto-percepción de clase de los docentes con la que le asignan a la mayoría de los alumnos de su escuela (Cuadro 7), se observa que a éstos les atribuyen una condición social, en promedio, más baja pero también "más dispersa", ya que adquieren mayor importancia relativa los segmentos más altos y más bajos de la pirámide.

En el año 2000 un 38,4\% asignó a sus alumnos una pertenencia de clase inferior a la propia; en el 2010, el porcentaje fue de 43,3. Más allá de que esta variación no resulta estadísticamente significativa, vale la pena apuntar una reflexión sobre 
consecuencias asociadas a la percepción de estar educando a los hijos de una clase social más baja que la que se percibe como propia. Dichas consecuencias van desde una posible sensación de pérdida de status por parte de los docentes (Drago et al., 2003) hasta el impacto negativo que esta imagen podría tener en aquello que los docentes eligen enseñar a los alumnos y en los resultados obtenidos por éstos (Calloids, 2008).

\section{Cuadro 7}

Auto-percepción de la clase social de los profesores y percepción de la clase social de la mayoría de los alumnos de su escuela.

Años 2000 y 2010. En Porcentaje.

\begin{tabular}{|l|rrr|rrr|}
\hline & \multicolumn{3}{|c|}{2000} & \multicolumn{3}{c|}{2010} \\
\hline Items de respuesta & Profesores & Alumnos & Dif. pp. & Profesores & Alumnos & Dif. pp. \\
\hline Alta & 0,0 & 0,6 & $-0,6$ & 0,9 & 0,4 & 0,5 \\
Media alta & 2,0 & 6,0 & $-4,0$ & 5,9 & 9,9 & $-4,0$ \\
Media & 54,3 & 36,4 & 17,9 & 64,6 & 38,0 & 26,6 \\
Media baja & 36,1 & 40,1 & $-4,0$ & 23,4 & 36,6 & $-13,2$ \\
Baja & 3,2 & 14,5 & $-11,3$ & 1,5 & 14,1 & $-12,6$ \\
No sabe & 4,4 & 2,3 & 2,1 & 3,8 & 1,0 & 2,8 \\
\hline Total & 100,0 & 100,0 & & 100,0 & 100,0 & \\
\hline
\end{tabular}

Fuente: Encuesta Nacional a Docentes 2000 y 2010 (IIPE-UnEsCO, Sede Regional Buenos Aires).

Para conocer la percepción sobre otra de las dimensiones frecuentemente utilizadas para caracterizar la estructura social como es la de la pobreza, se incluyó en los cuestionarios la pregunta "¿Cree que su hogar es pobre?". Como resulta evidente, se trata de un indicador sencillo para abordar el aspecto subjetivo, percibido, de la pobreza. En la medición del año 2000 los encuestados que contestaron afirmativamente representan el 13,5\%, mientras que en 2010 el porcentaje descendió a 8,3\% (cuadro siguiente).

En cuanto a las posibilidades que brinda la ocupación docente para la promoción social se propuso a los entrevistados comparar su situación económica actual con la de sus propios padres cuando eran niños como una forma de conocer percepciones sobre movilidad social inter-generacional.

Los profesores que manifestaron que su situación económica era "mejor" representaron el 33,4\% en el año 2000 y el 45,1\% en 2010 (Cuadro 9). En el otro extremo 
de la escala, el 31,6\% expresó estar "peor" en la primera medición y el 17,2\% en la segunda. Este núcleo de profesores que sigue experimentando una mengua en su situación económica con relación a sus padres tiene una importancia relativa mayor en el grupo intermedio de edad (30 a 39 años), entre los que se desempeñan en escuelas privadas, que tienen una forma de contratación precaria, que tienen título universitario y que pertenecen a hogares con menor nivel de ingresos per cápita.

\section{Cuadro 8}

Auto-percepción de pobreza de sus hogares por parte de los docentes Años 2000 y 2010. En Porcentaje.

\begin{tabular}{|l|rrrrr|}
\hline Items de respuesta & 2000 & 2010 & Dif. pp. & Dif \% & Var. signif. \\
\hline Pobre & 13,5 & 8,3 & $-5,2$ & $-38,5$ & Sí \\
No pobre & 74,0 & 78,7 & 4,7 & 6,3 & No \\
No sabe & 12,5 & 13,0 & 0,5 & 4,1 & No \\
\hline Total & 100,0 & 100,0 & 0,0 & 0,0 & \\
\hline
\end{tabular}

Fuente: Encuesta Nacional a Docentes 2000 y 2010 (IIPE-UNESCO, Sede Regional Buenos Aires).

\section{Cuadro 9}

Auto-percepción de pobreza de sus hogares por parte de los docentes Años 2000 y 2010. En Porcentaje.

\begin{tabular}{|l|c|c|c|c|c|}
\hline Items de respuesta & 2000 & 2010 & Dif. pp. & Dif \% & Var. Signif. \\
\hline Mejor & 33,4 & 45,1 & 11,7 & 35,2 & Sí \\
Igual & 30,6 & 31,7 & 1,1 & 3,6 & No \\
Peor & 31,6 & 17,2 & $-14,4$ & $-45,5$ & Sí \\
No sabe & 4,4 & 6,0 & 1,6 & 35,3 & No \\
\hline Total & 100,0 & 100,0 & 0,0 & 0,0 & \\
\hline
\end{tabular}

Fuente: Encuesta Nacional a Docentes 2000 y 2010 (IIPE-UNESCO, Sede Regional Buenos Aires).

Para finalizar, es necesario dejar apuntado que, además de la mejora relativa en los indicadores de nivel de actividad y empleo producida a lo largo de la década, se habría operado también un incremento en las remuneraciones docentes (Morduchowicz, 2009) impulsada por el compromiso conjunto asumido por la Nación y las 
Provincias a partir de la Ley de Financiamiento Educativo (Argentina, 2006a). En qué medida la mejora en la auto-percepción de la condición social de los profesores de secundaria está asociada con estos datos del contexto, y qué mediaciones operan en esta relación, es un tema que resta investigar.

\section{A manera de cierre}

Transcurrida la primera década del siglo, los y las docentes de secundaria de Argentina muestran algunos rasgos diferenciales respecto del inicio del mismo.

En lo que hace al trabajo en el aula, se manifiesta un incremento de las preocupaciones acarreadas por el vínculo con los alumnos y con los padres. Si se tiene en cuenta que, al mismo tiempo, ha perdido fuerza entre las respuestas el objetivo de transmitir conocimientos, puede conjeturarse que existe algún tipo de relación a explorar entre ambas variables, en tanto se desplazan parcialmente las preocupaciones desde funciones cognoscitivas hacia las de socialización e integración.

Más allá del incremento de las tensiones sentidas en la dimensión vincular, los docentes consignaron con sus respuestas una menor cantidad de problemas en la tarea cotidiana de enseñar, aunque persistiendo demandas asociadas a las exigencias laborales y el escaso tiempo para cumplirlas.

Por otra parte, sigue siendo considerable la proporción de profesores que espera dejar su trabajo en el aula en los próximos años, aunque una parte re-evaluaría esta aspiración si se le ofrecieran alternativas de mejora profesional sin dejar el espacio de trabajo cotidiano con los estudiantes.

Diez años después persiste una visión, en general, optimista acerca de lo que pueden ofrecer las TIC para el proceso de enseñanza-aprendizaje, aunque el modo en que esa visión se expresa en la actualidad parecería reconocer las dificultades para su incorporación y apropiación. Por otra parte, subsiste un núcleo de docentes que manifiesta desconfianza respecto a ciertos aspectos de la relación entre TIC y educación.

La mejora en la propia consideración social de los profesores es tal vez uno de los resultados más destacados que surge de la comparación de ambas encuestas. Es difícil sustraer esta conclusión de la recuperación de las variables del contexto social y económico que media entre los años 2000 y 2010 y del repunte relativo de los salarios docentes a partir de mediados de la década. Si bien no está al alcance de este análisis identificar los determinantes de aquella mejora, interesa dejarla consignada al menos como un contrapeso alentador frente a las crecientes dificultades y presiones que la tarea de educar impone hoy a las y los docentes. 


\section{Notas}

1. Las dimensiones de análisis son: características socio-demográficas del docente; características de su hogar; características de su escuela de inserción; condiciones de trabajo; aspectos pedagógicos; política educativa; formación docente; y tecnologías de información y comunicación.

2. La tasa de asistencia del grupo de edad de 12 a 17 años, para el total país, era del 87,4\% según el Censo Nacional de Población, Hogares y Viviendas del año 2001 (Argentina, 2011b). En el año 2009, para el mismo grupo de edad y en los 31 principales aglomerados urbanos (donde reside el 61,5\% de la población), la tasa alcanzaba el 92,3\% (SITEAL; IIPE-UNESCO; OEI, 2011).

3. En todos los cuadros del texto la columna con el encabezado "Dif. pp." presenta la variación en puntos porcentuales entre ambas mediciones; la columna "Dif. \%" consigna la variación porcentual entre los porcentajes de cada medición.; y la última columna ("Var. signif.".) indica si las diferencias observadas son estadísticamente significativas. Por otra parte, la sumatoria de los valores porcentuales de algunos cuadros excede el $100 \%$ debido a que los resultados se basan en una pregunta de respuesta múltiple.

\section{Referencias}

ARGENTINA. Base de datos del Censo Nacional de Población, Hogares y Viviendas del año 2001. Disponível em: <http://www.indec.mecon.ar/webcenso/index.asp>. Acesso em: 26 mayo 2011.

ARGENTINA. Ley Federal de Educación n. 24.195, del 14 de abril de 1993. Boletín Oficial de la República Argentina, Buenos Aires, n. 27.632, p. 1-5, 5 mayo 1993.

ARGENTINA. Ley de Financiamiento Educativo n. 26.075, del 21 de diciembre de 2005. Boletín Oficial de la República Argentina, Buenos Aires, n. 30.822, p. 1-4, 12 ene. 2006a.

ARGENTINA. Ley de Educación Nacional n. 26.206, del 14 de diciembre de 2006. Boletín Oficial de la República Argentina, Buenos Aires, n. 31.062, p. 1-10, 28 dic. 2006 b.

ARGENTINA. Ministerio de Economía y Finanzas Públicas. Información económica al día. Disponível em: <http://www.mecon.gov.ar/peconomica/basehome/infoeco. html>. Acesso em: 22 jul. 2011.

ARGENTINA. Ministerio de Educación, Ciencia y Tecnología de la Nación. Censo Nacional Docente 2004: resultados definitivos. Buenos Aires, 2006c.

ARGENTINA. Ministerio de Educación, Ciencia y Tecnología de la Nación. Consejo Federal de Educación. Resoluciones n. 31, 61, 84, 86, 88, 103, 135 y 136. Disponível em: <http://www.me.gov.ar/consejo/resoluciones/cf_resoluciones.html>. Acesso em: 22 jul. 2011.

AVALOS, B. et al. La profesión docente: temas y discusiones en la literatura internacional. Estudios Pedagógicos, Santiago, v. 36, n. 1, p. 235-263, 2010. Disponível em: <http://www.scielo.cl/pdf/estped/v36n1/art13.pdf> Acesso em: 4 mayo 2011. 
BURBULES, N.; CALLISTER, T. Educación: riesgos y promesas de las nuevas tecnologías de la información. Buenos Aires: Granica, 2006.

CALLOIDS, F. La lucha contra el fracaso escolar en los países desarrollados: ¿Cuál es el impacto de las políticas? In: Tenti Fanfani, E. (Org.). Nuevos temas en la agenda de política educativa. Buenos Aires: Siglo xxI, 2008. p. 145-172.

CEPEDA-CUERVO, E. et al. Intervalos de confianza e intervalos de credibilidad para una proporción. Revista Colombiana de Estadística, Bogotá, v. 31, n. 2, p. 221-228, dic. 2008. Disponível em: < http://www.kurims.kyoto-u.ac.jp/EMis/journals/RCE/V31/ bodyv31n2/v31n2a06CepedaEtAl.pdf > Acesso em : 28 abr. 2011.

CAPPELLACCI, I. et al. La transformación del nivel secundario (2006-2009). Buenos Aires: Ministerio de Educación de la Nación, 2011. Disponível em: <http://diniece. me.gov.ar/images/stories/diniece/publicaciones/boletin/9-Boletin-2011.pdf $>$. Acesso em: 12 jun. 2011.

DIRIE, C. El perfil de los docentes de Argentina: análisis realizado en base a los datos del Censo Nacional de Docentes 2004. Buenos Aires: Ministerio de Educación de la Nación, 2007. Disponível em:< http://diniece.me.gov.ar/images/stories/diniece/publicaciones/boletin/boletin4_sitio.pdf Acesso em:18 mayo 2011.

DONAIRE, R. Sobre la proletarización de los trabajadores intelectuales: un ejercicio comparativo a partir del caso de los docentes de Argentina. In: Neffea, J.; De La Garza, E.; MuÑIZ, L. (Org.). Trabajo, empleo, calificaciones profesionales, relaciones de trabajo e identidades laborales. Buenos Aires: Consejo Latinoamericano de Ciencias Sociales, 2009. p. 57-88.

DRAGO, R. et al. Attracting, developing and retaining effective teachers; Country Background Report for Italy. Paris: oECD, 2003. Disponível em: http://www.oecd.org/ dataoecd/54/7/17997702.pdf Acesso em: 4 mayo 2011.

DUSSEL, I. Impactos de los cambios en el contexto social y organización del oficio docente. In: Tenti Fanfani, E. (Org.). El oficio de docente: vocación, trabajo y profesión en el siglo xxi. Buenos Aires: Siglo xxI, 2006. p. 143-173.

ESTEVE, J. Identidad y desafíos de la condición docente. In: Tenti Fanfani, E. (Org.). El oficio de docente: vocación, trabajo y profesión en el siglo xxi. Buenos Aires: Siglo XXI, 2006. p. 19-69.

GERMANI, G. La clase media en la ciudad de Buenos Aires: estudio preliminar (1942). In: Mera, C.; Rebon, J. (Org.). Gino Germani: la sociedad en cuestión; antología comentada. Buenos Aires: Consejo Latinoamericano de Ciencias Sociales, 2010. p. 92-119. 
MEO, A. Identidades docentes en tiempos de cambios: entre el repliegue y la reinvención. London: The Economic and Social Research Council, 2010. Disponível em: <http:// www.esrc.ac.uk/my-esrc/grants/PTA-026-27-2053/outputs/Download/98869f10-f47743ef-b8fe-ccb9280b3b09> Acesso em: 2 abr. 2011.

MORDUCHOWICZ, A. Discusiones de economía de la educación. Buenos Aires: Losada, 2004.

MORDUCHOWICZ, A. Salarios docentes en la Provincia de Buenos Aires. Buenos Aires: Unidad Ejecutora Provincial; Dirección General de Cultura y Educación de la Provincia de Buenos Aires, 2009. (Mimeo.).

SITEAL; IIPE-UNESCO; OEI. Base de datos. Disponível em: <http://www.siteal.iipeoei.org/consulta_db_ext> Acesso em: 26 mayo 2011.

TENTI FANFANI, E. Profesionalización docente: consideraciones sociológicas. In: Tenti Fanfani, E. (Org.). El oficio de docente: vocación, trabajo y profesión en el siglo xxI. Buenos Aires: Siglo xxI, 2006. p. 119-142.

TENTI FANFANI, E. La condición docente. Buenos Aires: Siglo xxI, 2007.

TENTI FANFANI, E. Los que ponen el cuerpo: el profesor de secundaria en la Argentina actual. Educar em Revista, Curitiba, n. 1-Especial, p. 37-76, 2010. Disponível em: $<$ http://ojs.c3sl.ufpr.br/ojs2/index.php/educar/article/download/20464/13745> Acesso em: 12 jun. 2011.

TORRADO, S. Estrategias de desarrollo, estructura social y movilidad. In: ToRRADO, S. (Org.). Población y bienestar en la Argentina del primero al segundo centenario. Buenos Aires: Edhasa, 2007. p. 31-67.

Recebido em 20 de junho de 2011.

Aprovado em 3 de agosto de 2011. 